\title{
Constraints on the design of core-shell resonators of locally resonant acoustic metamaterials
}

\author{
Lionel Bos, ${ }^{1,2}$ Lyubov Lukyanova, ${ }^{3,4}$ and Régis Wunenburger ${ }^{1,2,5,6}$ \\ ${ }^{1}$ Univ. Bordeaux, LOMA, UMR 5798, F-33405 Talence, France \\ ${ }^{2}$ CNRS, LOMA, UMR 5798, F-33400 Talence, France \\ ${ }^{3}$ CNRS, CRPP, UPR 8641, F-33600 Pessac, France \\ ${ }^{4}$ Univ. Bordeaux, CRPP, UPR 8641, F-33600 Pessac, France \\ ${ }^{5}$ UPMC Univ Paris 06, UMR 7190, Institut Jean Le Rond d'Alembert, F-75005 Paris, France \\ ${ }^{6}$ CNRS, UMR 7190, Institut Jean Le Rond d'Alembert, F-75005 Paris, France \\ (Received 22 March 2012; revised manuscript received 4 October 2012; published 16 November 2012)
}

\begin{abstract}
We perform a parametric study of the analytic model of Liu et al. [Z. Liu et al., Phys. Rev. B 71, 014103 (2005)] describing the mechanical response of a core-shell particle to an acoustic excitation in order to help in selecting the constitutive materials and in designing innovative processes of fabrication of downsized core-shell resonators, which are key constituents of locally resonant acoustic metamaterials. We show that the value of the first Lamé coefficient of the material constituting the shell has no marked influence on the value of the resonance frequency of the core-shell resonator, that is, it does not necessarily need to be small for satisfying the condition of subwavelength resonator dimension at resonance. Moreover, we show that the larger the density contrast between the core and the shell and the thinner the shell, the broader is the frequency band over which the effective density of the resonator suspension is negative, but that it is practically useless to decrease the dimensionless shell thickness below 0.6. Finally, we show that the dissipation is also less perceptible the thinner is the shell and the larger is the density contrast. The effect of the density contrast between the core and the shell and of the dissipation on the resonance width are explained by comparing with the harmonic oscillator and the mass-in-mass $1 \mathrm{D}$ lattice.
\end{abstract}

DOI: 10.1103/PhysRevB.86.184107

PACS number(s): 43.20.+g, 43.35.+d, 43.40.+s

\section{INTRODUCTION}

Since a decade, an intense worldwide research effort has been devoted to the design and fabrication of artificial materials allowing for renewed strategies of control of the propagation of acoustic waves and resulting in flat lenses, subwavelength imaging, improved sound isolation and filtering, cloaking, etc. Two main classes of composite materials have been early identified for achieving these goals, sonic crystals (SC), and locally resonant acoustic metamaterials (LRAM), whose unique behavior is due to embedded subwavelength mechanical resonators. On the one hand, sonic crystals have already allowed to achieve negative refraction in the ultrasonic frequency range in two-dimensional (2D) $)^{1-3}$ and three-dimensional (3D) configurations. ${ }^{4}$ On the other hand, while in the audible range several successful realizations have been reported in $1 \mathrm{D},{ }^{5} 2 \mathrm{D}, 6$ and $3 \mathrm{D},{ }^{7,8}$ in the ultrasonic frequency range only $1 \mathrm{D}^{9}$ and $2 \mathrm{D}$ LRAM $^{10,11}$ could be achieved. However, LRAM constitute a promising class of acoustic materials since their unique properties are not intrinsically associated with any periodic order at the wavelength scale, contrary to SC, but to the resonant nature of their subwavelength microstructure. This permits to obtain pronounced effects using extremely thin samples compared to the wavelength, ${ }^{7,8}$ even using a single layer of resonators, ${ }^{12-15}$ so to foresee compact acoustical devices. This justifies further research efforts on LRAM. Remarkably, all the mechanical resonators involved in these LRAM have been manufactured. The difficulty of downscaling such fabrication processes combined with the subwavelength dimensions imposed to the resonators probably explains why the working frequency range of 3D LRAM have been up to now restricted to the audible range. The miniaturization of the mechanical resonators is presently an issue for the achievement of 3D, LRAM working in the ultrasonic frequency ranges employed in nondestructive testing, underwater detection, and medical imaging. Another already pointed issue is the increase of dissipation with frequency, which is known to damp the resonances and is consequently expected to prevent from obtaining the targeted effects of LRAM.

Among the variety of resonators, which have been theoretically proposed for constituting LRAM and partly experimentally achieved, ${ }^{5-10,14,16-25}$ two kinds of resonators have a sufficiently simple structure to be potentially downsized and to produce 3D effects: air bubbles and core-shell particles. ${ }^{7}$ Their combination has even been predicted to result in negative refraction. ${ }^{20}$ On the one hand, the acoustic properties of air bubbles and of bubbly media are rather well documented, ${ }^{26,27}$ and the resonant behavior of micrometer-sized air bubbles has already been exploited at frequencies of the order of the $\mathrm{MHz} .{ }^{15}$ On the other hand, although core-shell particles have demonstrated their efficiency in the audible frequency range ${ }^{7}$ and have been the subject of mechanical ${ }^{28}$ and acoustical modelings, ${ }^{20,29}$ neither their downscaling nor the effect of dissipation on their resonance has been studied. This is precisely the purpose of this work, which exploits the mechanical model of core-shell resonator proposed in Ref. 28 to investigate the influence of the dimensions as well as of the densities and visco-elastic properties of the constitutive materials on the resonance of core-shell particles. We believe that this study should be useful for selecting the constitutive materials and designing innovative processes of fabrication of downsized core-shell resonators.

Our article is organized as follows: in Sec. II, we briefly recall the geometry and the main ingredients of the model of core-shell resonator presented in Ref. 28 that we will 
use throughout this work and we propose an elementary scaling analysis of the resonance. In Sec. III, we study the dependence of the resonance frequency of the core-shell resonator versus its dimensions and the physical properties of its constitutive materials in absence of dissipation. In Sec. IV, we study how the width of the resonances of the core motion and of the effective density of the suspension depends on the same quantities and we compare our findings with the harmonic oscillator and the mass-in-mass 1D lattice [sketched in Fig. 1(b)], which mimics the core-shell suspension and is a generic medium exhibiting negative effective density. ${ }^{30,31}$ In the following, the frequency band over which the effective density of a suspension of core-shell resonators is predicted to be negative is called the negative effective density frequency band (NEDFB). The conclusions of Sec. IV allow us to enlighten in Sec. $\mathrm{V}$ the observed dependence of the NEDFB versus the same quantities. Finally, in Sec. VI, we study and explain how dissipation influences the width of the NEDFB.

\section{MAIN ASSUMPTIONS OF THE MODEL AND SCALING ANALYSIS}

The model presented in Ref. 28 describes the motion of a suspension of core-shell particles under the action of a plane, harmonic, longitudinal, incident wave. A core-shell resonator is composed of a rigid, spherical core coated with a spherical shell made of a "soft" material, the whole being embedded in a rigid matrix, see Fig. 1(a). The core is required to be rigid enough compared to the shell so that its deformation in the frequency regime of interest can be ignored regarding the shell deformation. The matrix is required to be rigid enough compared to the shell so that the mechanical effect of a local resonator is not sensitive to its nearest neighbor and so that its motion in contact with the resonator can be considered as homogeneous. Acoustic interactions between the resonators are not considered, an assumption a priori valid in the limit of infinitely small volume fractions and of infinitely large wavelength (compared to the core-shell particle size) and favored by the rigidity of the matrix. In this frame, the description of the dynamics of a core-shell particle is reduced to the resolution of the propagation of

(a)

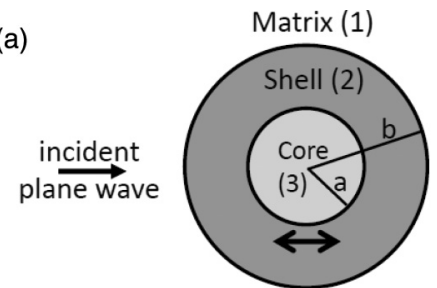

(b)

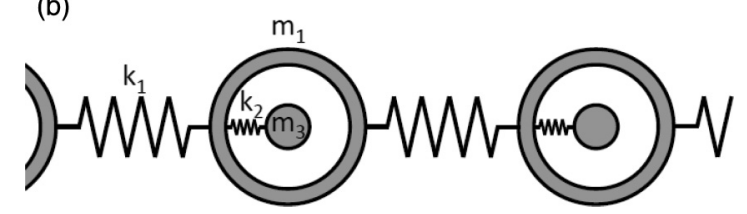

FIG. 1. (a) Sketch of the hard core-soft shell resonator, as modeled in Ref. 28. (b) Mass-in-mass lattice mimicking the core-shell suspension. coupled longitudinal and transverse waves within the shell, while both the matrix and the core oscillate harmonically, each as a whole. The model predicts the displacement field within the shell coupled with the motion of the core resulting from the vibration of the matrix. Qualitatively, the core is mobile within the cavity but its motion is limited by the restoring force exerted by the deformed shell. Since the core has inertia and the deformed shell acts on the core as a spring, the motion of the core center of mass parallel to the direction of the incident wave displays a resonance. The simplifying assumptions of this model correspond well to the characteristic features of the core-shell based LRAM which has been successfully implemented by the same authors ${ }^{7}$ and to the experimental conditions of its implementation. Furthermore, this simplified model is aimed at catching the physics of core-shell resonators under the assumptions of hard core and matrix, since it follows a full acoustic modeling of the same LRAM with periodic order based on the matrix scattering theory. ${ }^{29}$ Note that only this model enables to study the influence of dissipation on the resonance of such core-shell resonators, a fact that we will exploit in this study.

The properties of the matrix, shell, and core being labeled by index 1, 2 and 3, respectively, [see Fig. 1(a)], and $\rho_{i}$, $i=1-3$ being the densities of the materials constituting the three media, the effective density $\rho_{\text {eff }}$ of a suspension of core-shell resonators with volume fraction $\phi$, irradiated by a longitudinal, plane, harmonic wave with frequency $f=\frac{\omega}{2 \pi}$, has the following form: ${ }^{28}$

$$
\rho_{\mathrm{eff}}=(1-\phi) \rho_{1}+\phi \rho_{r}(\omega),
$$

where $\rho_{r}(\omega)$, which is the effective dynamic density of the resonator, takes account of its internal resonant dynamics and is defined as

$$
\rho_{r} V_{r} \mathbf{a}_{r}=\mathbf{F}_{r},
$$

where $\mathbf{F}_{r}$ is the harmonic force exerted by the matrix on the resonator, $V_{r}$ is the resonator volume, and $\mathbf{a}_{r}$ is the harmonic acceleration of the matrix in contact with the resonator.

In absence of dissipation, at resonance, the effective density $\rho_{\text {eff }}$ becomes infinite. Although $\rho_{\text {eff }}$ actually exhibits several resonances, in this work, we focus on the resonance with the lowest frequency ("first resonance"). It is indeed the resonance on which the effect of dissipation is expected to be the smallest, as inferred from Ref. 28. A typical curve of variation of the dimensionless effective density $\tilde{\rho}_{\text {eff }}=\rho_{\text {eff }} / \rho_{0}$ versus $f$ around its first resonance is shown in Fig. 2, where $\rho_{0}$ is the static mean density of the suspension of core-shell resonators. For simplicity purpose, the frequency of the first resonance is hereafter called the resonance frequency and is noted $f_{\text {res }}$.

Given the simplifying assumptions of the model, $f_{\text {res }} a$ priori only depends on the densities of the core and of the shell $\rho_{3}$ and $\rho_{2}$, on the core radius $a$ and shell outer radius $b$, and on the viscoelastic properties of the shell, which are characterized by the complex Lamé coefficients $\lambda_{2}$ and $\mu_{2}$ ( $\mu_{2}$ identifies with the shear elastic modulus of the shell). It is indeed assumed in the model presented in Ref. 28 that the resonators are not acoustically coupled, so their resonance, thus $f_{\text {res }}$, is independent of the resonators volume fraction $\phi$ and of the matrix density $\rho_{1}$. Neglecting here dissipation, i.e., considering $\lambda_{2}$ and $\mu_{2}=\mu_{2}^{\prime}$ as real, dimensional analysis 


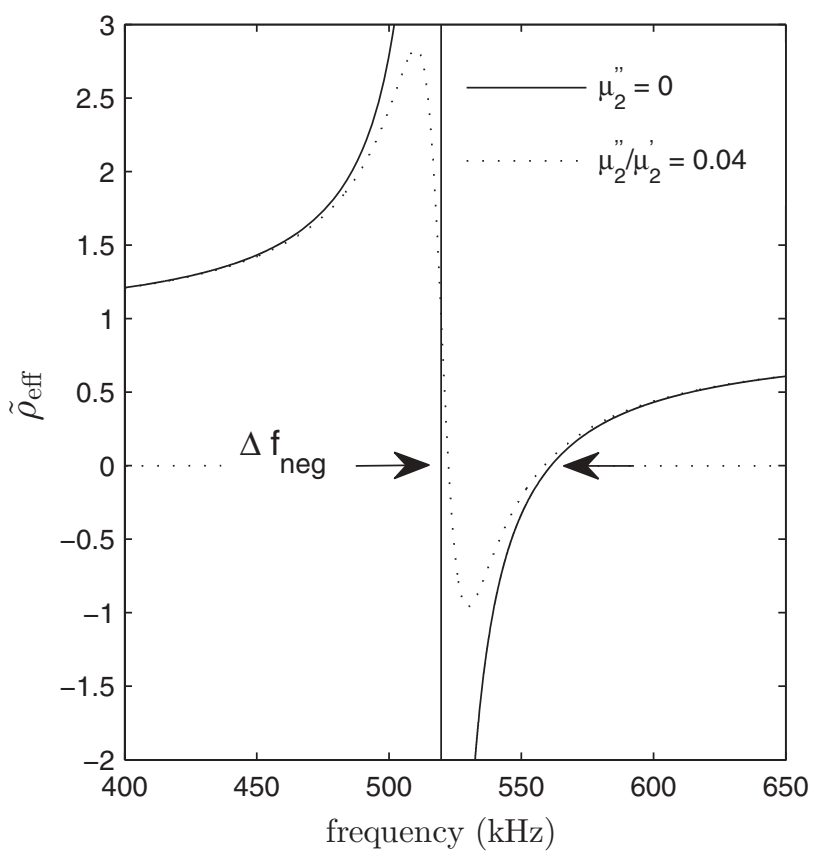

FIG. 2. Frequency dependence of the dimensionless effective density $\tilde{\rho}_{\text {eff }}$ of a suspension of core-shell resonators in the vicinity of the lowest resonance frequency $f_{\text {res }}$ (called in this work the resonance frequency), the without dissipation (solid curve) and with dissipation (dotted curve). $\rho_{1}=1000 \mathrm{~kg} . \mathrm{m}^{-3}, \rho_{2}=1090 \mathrm{~kg} \mathrm{~m}^{-3}$, $\rho_{3}=8860 \mathrm{~kg} \mathrm{~m}^{-3}, \lambda_{2}=10^{9} \mathrm{~Pa}, \mu_{2}^{\prime}=2.5 \times 10^{6} \mathrm{~Pa}, a=50 \mu \mathrm{m}$, $b=75 \mu \mathrm{m}$, and $\phi=0.1$.

allows to state that

$$
\tilde{f}_{\mathrm{res}}=\frac{f_{\text {res }}}{\sqrt{\frac{\mu_{2}^{\prime}}{\rho_{3} a^{2}}}}=\mathfrak{F}\left(\frac{\mu_{2}^{\prime}}{\lambda_{2}}, \varepsilon, \Delta \tilde{\rho}\right),
$$

where $\varepsilon=\frac{b-a}{a}$ is the dimensionless shell thickness, $\Delta \tilde{\rho}=$ $\frac{\rho_{3}-\rho_{2}}{\rho_{2}}$ is the dimensionless density contrast, and $\mathfrak{F}$ is an unknown function.

As shown hereafter, the resonance is marked for large density contrasts, $\Delta \tilde{\rho} \gg 1$. Besides, the hypothesis of rigid core and matrix is satisfied when the core and the matrix are made of hard solids and conversely the shell is made of a soft material, which often satisfies $\mu_{2}^{\prime} \ll \lambda_{2}$. In these limits, the oscillating mass $m$ is mainly the mass of the core, which scales as $m \propto \rho_{3} a^{3}$, and the density of the shell remains homogeneous during its shear associated to the core vibration. Denoting $x$ the amplitude of the harmonic oscillation of the core, the strain exerted by the core on the shell scales as $x /(b-a)$, so the shear stress exerted by the shell on the core scales as $\mu_{2}^{\prime} \frac{x}{b-a}$, so the net restoring force $F$ exerted by the shell on the whole surface of the core of area $\propto a^{2}$ scales as $F \propto a^{2} \mu_{2}^{\prime} \frac{x}{b-a}$ and the associated spring constant $k$ scales as $k=F / x \propto \frac{\mu_{2}^{\prime} a^{2}}{b-a}$. Finally, the resonance frequency, which scales as $\sqrt{\frac{k}{m}}$, is expected to scale as

$$
f_{\mathrm{res}} \propto \sqrt{\frac{\mu_{2}^{\prime}}{\rho_{3} a^{2}}} \varepsilon^{-1 / 2} .
$$

Although this scaling law has to be confronted to the analytical model of Ref. 28, it justifies our choice for the dimensionless quantities appearing in Eq. (3).

\section{RESONANCE FREQUENCY}

In this section, we study how the resonance frequency of the core-shell resonator depends on the dimensions and on the physical properties of its constitutive materials in absence of dissipation.

First, we study the dependence of $f_{\text {res }}$ versus the Lamé coefficients of the material constituting the shell. The variation of $\tilde{f}_{\text {res }}$ versus $\mu_{2}^{\prime} / \lambda_{2}$ for $\varepsilon=0.5$ and $\Delta \tilde{\rho}=8$, shown in Fig. 3, evidences that $\tilde{f}_{\text {res }}$ varies only by a factor of 2 between its asymptotic, upper value when $\mu_{2}^{\prime} / \lambda_{2} \rightarrow 0$ (soft solids) and its lower value when $\mu_{2}^{\prime} / \lambda_{2}$ is of the order to unity (common hard solids). The same conclusions can be drawn for other values of $\Delta \tilde{\rho}$ and of $\varepsilon$. This demonstrates that $\lambda_{2}$ has no marked influence on the resonance frequency of the resonator. This can be explained by noting that, since in this model the core moves within the shell without changing its own volume, the shell is essentially sheared, so the stiffness of the resonator is mainly associated with the resistance of the shell to shear and not to compression. Furthermore, once $\lambda_{2}$ and $\mu_{2}^{\prime}$ are sufficiently small compared to $\lambda_{i}$ and $\mu_{i}, i=1,3$, for allowing the hypothesis of rigid core and matrix to be satisfied, this model shows that the only mechanical parameter on which the resonance frequency essentially depends is $\mu_{2}^{\prime}$. Since arbitrarily small values of $\mu_{2}^{\prime}$ can be obtained using soft solids like polymers or gels, the resonance frequency can be lowered down at will in order to satisfy the condition of subwavelength size of the resonators at resonance, independently of the value of $\lambda_{2}$. Since the values of $\lambda$ of almost all solids, including the

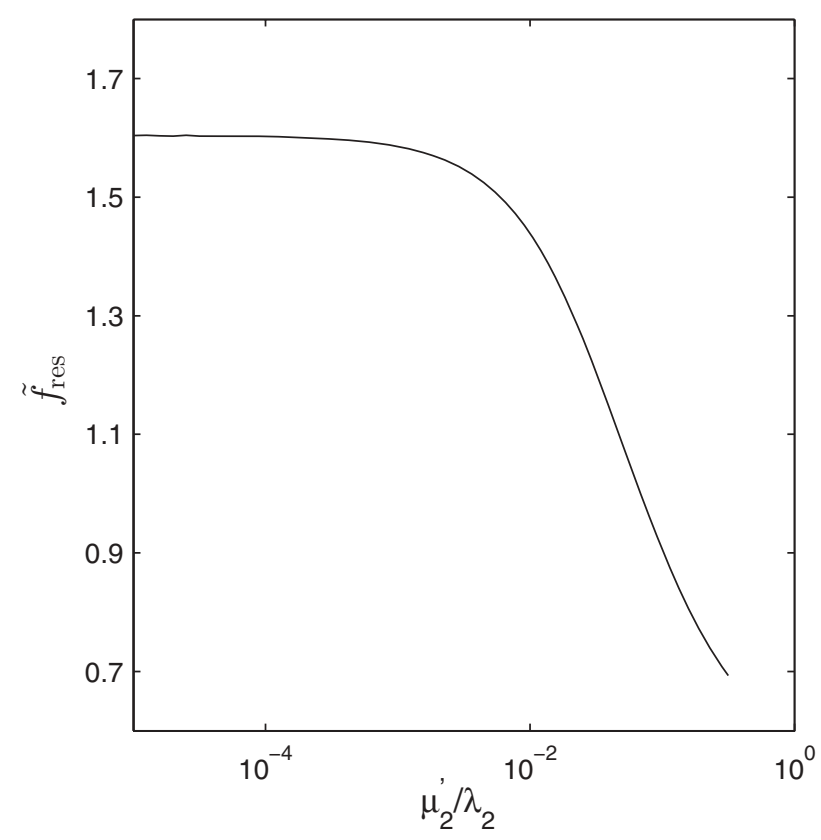

FIG. 3. Variation of the dimensionless resonance frequency of the core-shell resonator $\tilde{f}_{\text {res }}$ vs the ratio of the second $\left(\mu_{2}^{\prime}\right)$ to first $\left(\lambda_{2}\right)$ Lamé coefficients of the material constituting the shell in absence of dissipation. $\Delta \tilde{\rho}=8$ and $\varepsilon=0.5$. 


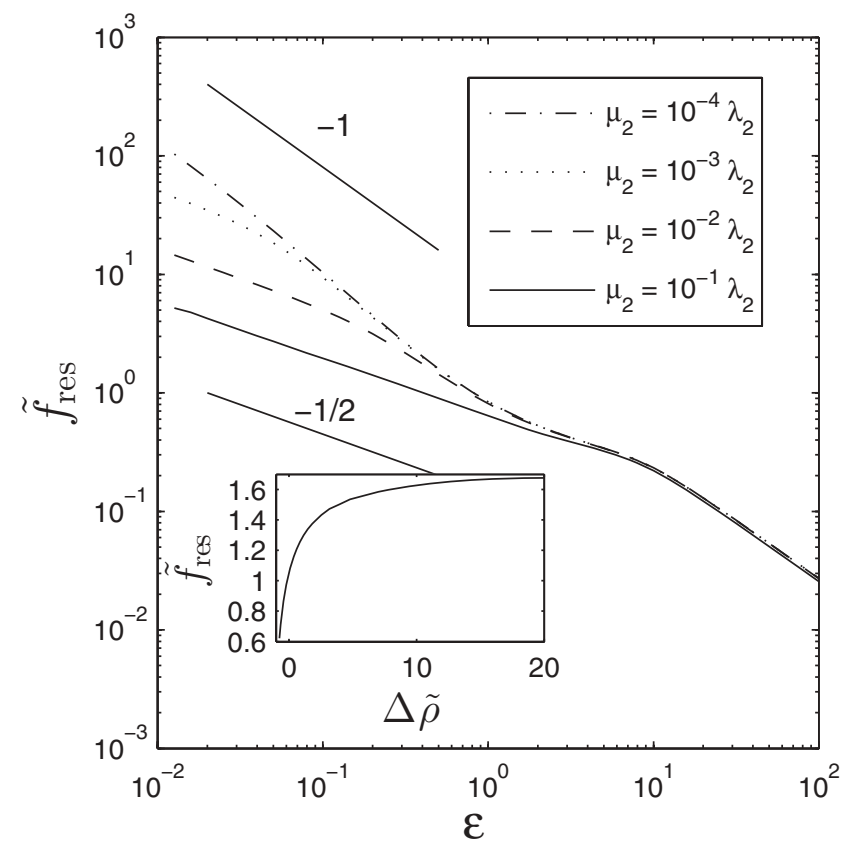

FIG. 4. Variation of the dimensionless resonance frequency of the core-shell resonator $\tilde{f}_{\text {res }}$ vs the dimensionless shell thickness $\varepsilon$ in absence of dissipation. $\Delta \tilde{\rho}=8$. (Inset) Variation of $\tilde{f}_{\text {res }}$ versus $\Delta \tilde{\rho}$ in absence of dissipation. $\varepsilon=0.5$ and $\mu_{2}^{\prime} / \lambda_{2}=10^{-4}$.

soft ones, are of the order of or larger than $1 \mathrm{GPa}$ (as it can be inferred from the values of the celerity $c_{L}=\sqrt{(\lambda+2 \mu) / \rho}$ of longitudinal waves in solids, including soft ones, which range from $10^{3} \mathrm{~m} \mathrm{~s}^{-1}$ to $10^{4} \mathrm{~m} \mathrm{~s}^{-1}$, and of their density which range from $10^{3} \mathrm{~kg} \mathrm{~m}^{-3}$ to $10^{4} \mathrm{~kg} \mathrm{~m}^{-3}$ ), the fact that the value of $\lambda_{2}$ does not need to be very small for obtaining small values of $f_{\text {res }}$ enlarges the range of usable materials for constituting the shell.

We now study the dependence of $\tilde{f}_{\text {res }}$ versus the dimensionless shell thickness $\varepsilon$. The variations of $\tilde{f}_{\text {res }}$ versus $\varepsilon$ for several values of $\mu_{2}^{\prime} / \lambda_{2}$ ranging from $10^{-4}$ to $10^{-1}$, shown in Fig. 4 , evidence that in the $\varepsilon<1$ range which, as shown hereafter, is the relevant one, $\tilde{f}_{\text {res }}$ scales as $\varepsilon^{-1 / 2}$ when $\mu_{2}^{\prime} / \lambda_{2} \simeq 1$ (hard solid), where it scales differently, namely, as $\varepsilon^{-1}$ when $\mu_{2}^{\prime} / \lambda_{2} \ll 1$ (soft solid).

Finally, we study the dependence of $\tilde{f}_{\text {res }}$ versus the dimensionless density ratio $\Delta \tilde{\rho}$. The variation of $\tilde{f}_{\text {res }}$ versus $\Delta \tilde{\rho}$ ranging from -0.2 (corresponding to a core made of a light organic phase and encapsulated in a shell made of an aqueous gel) to 20 (corresponding to a platinum core encapsulated in an organic or aqueous phase), shown in the inset of Fig. 4, evidence that $\tilde{f}_{\text {res }}$ does not significantly depend neither on the sign nor on the value of the density ratio in a range of realistic values.

These results lead us to state that in the case of soft $\left(\mu_{2}^{\prime} \ll \lambda_{2}\right)$ thin shells $(b-a<a)$, which, as shown hereafter, is the most interesting case, $f_{\text {res }}$ approximately satisfies the following scaling law:

$$
f_{\mathrm{res}} \simeq \sqrt{\frac{\mu_{2}^{\prime}}{\rho_{3}(b-a)^{2}}},
$$

which can be used as an abacus for designing the core-shell resonators. Note that this scaling law is in disagreement with the prediction Eq. (4) of the scaling-law model. This could be ascribed to the roughness of the assumption made in the scaling-law model that the same shear is exerted on the whole surface of the core.

In the case of hard $\left(\mu_{2}^{\prime} \simeq \lambda_{2}\right)$ thin shells $(\varepsilon<1), \tilde{f}_{\text {res }}$ approximately satisfies the following scaling law:

$$
f_{\text {res }} \simeq \sqrt{\frac{\mu_{2}^{\prime}}{\rho_{3} a^{2}}} \varepsilon^{-1 / 2} .
$$

Note that this scaling law is in agreement with the prediction Eq. (4) of the scaling-law model, although the latter does not take into account the resistance of the shell to compression.

\section{RESONANCE WIDTH}

In this section, we study how the widths of the resonances of the core motion and of the effective density of the suspension, on which the obtention of the targeted negative effective density property relies, depend on the resonator dimensions and on the physical properties of its constitutive materials. In addition, for the purpose of physical interpretation, we compare our results with the properties of the mass-in-mass 1D lattice. In the following, we define the dimensionless effective density deviation $\Delta \tilde{\rho}_{\text {eff }}$ as $\Delta \tilde{\rho}_{\text {eff }}=\frac{\rho_{\text {eff }}-\rho_{0}}{\rho_{0}}=\tilde{\rho}_{\text {eff }}-1$, and the dimensionless core displacement amplitude $\tilde{x}$ as $x / X$, where $X$ is the amplitude of the harmonic displacement of the matrix. Since absence of dissipation is assumed, the core displacement amplitude and the effective density are both infinite at resonance. Therefore the resonance width of $\tilde{x}$ (respectively, $\Delta \tilde{\rho}_{\text {eff }}$ ) cannot be defined here as the full width at half of its maximum. It is rather defined as the frequency bandwidth over which $\tilde{x}$ (respectively, $\left|\Delta \tilde{\rho}_{\text {eff }}\right|$ ) is larger than a given threshold $T$, say 10 , and it is labeled $\Delta f_{\tilde{x}>10}$ (respectively, $\Delta f_{\left|\Delta \tilde{\rho}_{\text {eff }}\right|>10}$ ). We note that a harmonic oscillator forced with fixed displacement amplitude $X$ is such that $\Delta \tilde{f}_{\tilde{x}>T}=\frac{\Delta f_{\tilde{x}>T}}{f_{\text {res }}} \simeq T^{-1}$ in the $T \gg 1$ limit. Its displacement amplitude $x$ indeed satisfies $\tilde{x}=x / X=\left(1-\tilde{\omega}^{2}\right)^{-1}$, where $\tilde{\omega}=\omega / \omega_{\text {res }}$, with $\omega_{\text {res }}=\sqrt{k_{2} / m_{3}}, k_{2}$ being the spring stiffness, and $m_{3}$ the mass of the oscillator.

First, we focus on the resonance of the core motion, i.e., on the properties of $\tilde{x}$. Dimensional analysis allows to state that $\Delta \tilde{f}_{\tilde{x}>10}$ is a function of $\frac{\mu_{2}^{\prime}}{\lambda_{2}}, \varepsilon$, and $\Delta \tilde{\rho}$ only (as already pointed in Sec. II, it is assumed in the model of Ref. 28 that the resonators are not acoustically coupled, so their resonance is independent of $\phi$ and of $\rho_{1}$ ). In the following, we restrict our numerical investigation to the $\frac{\mu_{2}^{\prime}}{\lambda_{2}} \ll 1$ limit in which the condition of subwavelength size of the resonators at resonance can be easily satisfied, see Sec. II above. The variations of $\Delta \tilde{f}_{\tilde{x}>10}$ versus $\Delta \tilde{\rho}$ ranging from $10^{-2}$ to $10^{2}$ for several values of $\varepsilon$ shown in Fig. 5 , evidence that $\Delta \tilde{f}_{\tilde{x}>10}$ increases proportionally to $\Delta \tilde{\rho}$ in the $\Delta \tilde{\rho} \ll 1$ limit, then saturates at $10^{-1}$ beyond $\Delta \tilde{\rho}>20$. Noticeably, for $\varepsilon \simeq 1$, the variations of $\Delta \tilde{f}_{\tilde{x}>10}$ are found to be well approximated by the function $10^{-1}\left(\rho_{3}-\rho_{2}\right) /\left(\rho_{3}+\rho_{2}\right)=10^{-1} \Delta \tilde{\rho} /(2+\Delta \tilde{\rho})$.

In order to understand this behavior, we consider the mass-in-mass 1D lattice schematized in Fig. 1, which mimics the core-shell suspension. ${ }^{30,31}$ In this model, the rigid core 


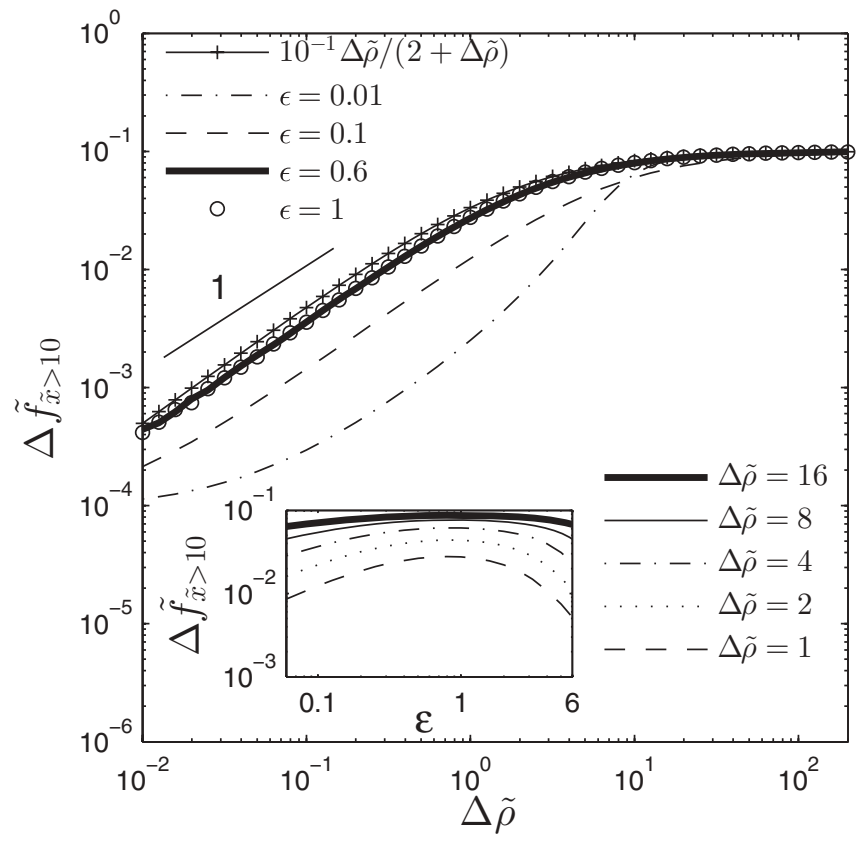

FIG. 5. Variations of the dimensionless frequency bandwidth over which the dimensionless displacement amplitude of the core $\tilde{x}$ is larger than $10, \Delta \tilde{f}_{\tilde{x}>10}$, vs the dimensionless density contrast $\Delta \tilde{\rho}$ for several values of dimensionless shell thickness $\varepsilon$ in absence of dissipation. $\mu_{2}^{\prime} / \lambda_{2}=10^{-4}$. (Inset) Variations of $\Delta \tilde{f}_{\tilde{x}>10}$ vs $\varepsilon$ for several realistic values of $\Delta \tilde{\rho}$.

is represented by the mass $m_{3}$, while the elastic shell is represented by the spring with stiffness $k_{2}$. The spring with stiffness $k_{1}$ and the mass $m_{1}$ represent the matrix. A straightforward discrete model of this lattice shows that $\tilde{x}=\left(1-\tilde{\omega}^{2}\right)^{-1} \cdot{ }^{32}$ This implies that, in the same manner as for the harmonic oscillator, $\Delta \tilde{f}_{\tilde{x}>10} \simeq 10^{-1}$ for the mass-in-mass lattice. The $\left(\rho_{3}-\rho_{2}\right) /\left(\rho_{3}+\rho_{2}\right)$ factor differentiating the $\tilde{x}$ resonances of the mass-in-mass lattice and of the core-shell is a signature of the inertia of the shell, which is not taken into account in the mass-in-mass lattice model (in this model, the shell is represented by its stiffness only). This factor can be qualitatively explained in the following manner: when the core-shell particle is accelerated by the matrix, the accelerated shell exerts on the core stresses whose resultant force, $-(4 \pi / 3) a^{3} \rho_{2} \mathbf{a}_{r}$, adds to the force due to its deformation by the core (it is equivalent to buoyancy in fluids). Thus the core moves as if its effective mass was $(4 \pi / 3) a^{3}\left(\rho_{3}-\rho_{2}\right)$. Thus the less the density contrast the less marked is the resonance, so the smaller is $\Delta \tilde{f}_{\tilde{x}>10}$. Accordingly, when the shell inertia is much smaller than the core inertia of $\Delta \tilde{\tilde{f}}_{\tilde{x}>10}$ saturates at the same value as for the mass-in-mass lattice in which the shell has no inertia.

Besides, the variations of $\Delta \tilde{f}_{\tilde{x}>10}$ versus $\varepsilon$ ranging from 0.06 to 6 for several realistic values of $\Delta \tilde{\rho}$ shown in insert of Fig. 5, evidence that $\Delta \tilde{f}_{\tilde{x}>10}$ is maximal at $\varepsilon \simeq 0.8$ whatever $\Delta \tilde{\rho}$ and that this maximum increases with $\Delta \tilde{\rho}$.

We now study the consequence of the core motion resonance on the effective density of the core-shell suspension $\rho_{\text {eff }}$. The behavior of $\rho_{\text {eff }}$ is indeed directly determined by the behavior of $x$ through Eq. (36) of Ref. 28, which is of the form $\rho_{\text {eff }}=\rho_{1}(1-\phi)+\rho_{2} \phi\left[g_{1}(\omega)-g_{2}(\omega) \frac{a}{b} \frac{x}{X}\right]$, where

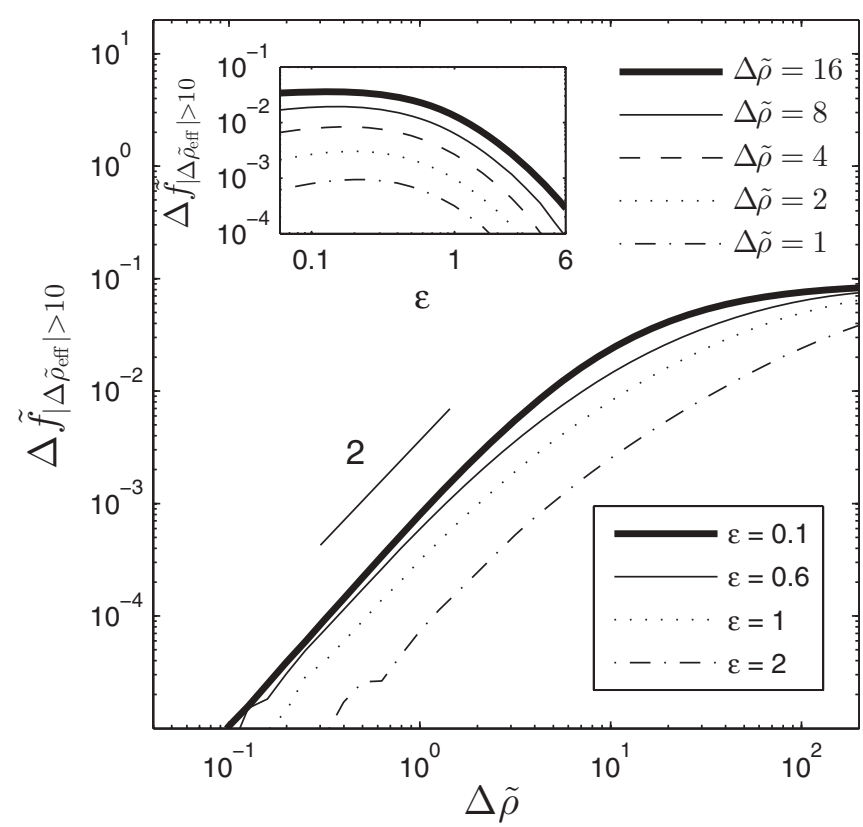

FIG. 6. Variations of the dimensionless frequency bandwidth over which the dimensionless effective density deviation $\Delta \tilde{\rho}_{\text {eff }}$ is larger than $10, \Delta \tilde{f}_{\left|\Delta \tilde{\rho}_{\text {eff }}\right|>10}$, vs the dimensionless density contrast $\Delta \tilde{\rho}$ for several values of dimensionless shell thickness $\varepsilon$ in absence of dissipation. $\mu_{2}^{\prime} / \lambda_{2}=10^{-4}$. (Inset) Variations of $\Delta \tilde{f}_{\left|\Delta \tilde{\rho}_{\text {eff }}\right|>10}$ vs $\varepsilon$ for several realistic values of $\Delta \tilde{\rho}$.

$g_{1}$ and $g_{2}$ are regular functions of $\omega$. In particular, the resonance frequency of the core motion coincides with $f_{\text {res }}$. Dimensional analysis allows to state that $\Delta \tilde{f}_{\left|\Delta \tilde{\rho}_{\text {eff }}\right|>10}=\frac{\Delta f_{\left|\Delta \tilde{\rho}_{\text {eff }}\right|>10}}{f_{\text {res }}}$ is a function of $\frac{\mu_{2}^{\prime}}{\lambda_{2}}, \varepsilon, \Delta \tilde{\rho}, \phi$, and $\rho_{2} / \rho_{1}$. Since the model of Ref. 28 neglects the acoustic interactions between the particles, strictly speaking it is only valid in the limit of vanishing values of $\phi$. This is why we will not perform the parametric study of the resonator behavior as a function of $\phi$ and we definitely fix $\phi=0.1$ in the following.

In addition, we definitely fix $\rho_{2} / \rho_{1}=1$, since the effect of $\rho_{1}$ on $\rho_{\text {eff }}$ is obvious from Eq. (1) ( $g_{1}$ and $g_{2}$ do not depend on $\left.\rho_{1}\right)$. The variations of $\Delta \tilde{f}_{\left|\Delta \tilde{\rho}_{\text {eff }}\right|>10}$ versus $\Delta \tilde{\rho}$ ranging from $10^{-2}$ to $10^{2}$ for several values of $\varepsilon$, shown in Fig. 6, evidence that $\Delta \tilde{f}_{\left|\Delta \tilde{\rho}_{\text {eff }}\right|>10}$ increases proportionally to $(\Delta \tilde{\rho})^{2}$ in the $\Delta \tilde{\rho} \ll 1$ limit, then saturates at $10^{-1}$ beyond $\Delta \tilde{\rho}>80$. The quadratic dependence of $\Delta \tilde{f}_{\left|\Delta \tilde{\rho}_{\text {eff }}\right|>10}$ versus $\Delta \tilde{\rho}$ can be explained by noting that, when the core moves to the left [see Fig. 1(a)], the matter of the shell moves to the right because of incompressibility of the shell in the $\frac{\mu_{2}^{\prime}}{\lambda_{2}} \ll$ 1 limit. As a consequence, the effect of the core motion $\tilde{x}$ on the mass distribution within the particle, hence on $\rho_{\text {eff }}$, is proportional to the density difference between the core and the shell. Consequently, it is expected that $\Delta \tilde{f}_{\left|\Delta \tilde{\rho}_{\text {eff }}\right|>10} \propto \Delta \tilde{\rho} \times$ $\Delta \tilde{f}_{\tilde{x}>10} \propto \Delta \tilde{\rho}^{2}$, in agreement with our numerical results.

Considering the mass-in-mass lattice, whose effective mass $m_{\text {eff }}$ is such that $\Delta \tilde{m}_{\text {eff }}=\left(m_{\text {eff }}-m_{0}\right) / m_{0}=$ $\left(m_{3} / m_{0}\right) \tilde{\omega}^{2} /\left(1-\tilde{\omega}^{2}\right)$, where $m_{0}=m_{1}+m_{3}$, we note that $\Delta \tilde{f}_{\left|\Delta \tilde{m}_{\text {eff }}\right|>10}=\frac{\Delta f_{\left|\Delta \tilde{m}_{\text {eff }}\right|>10}}{f_{\text {res }}} \simeq 10^{-1}$, which is precisely the asymptotic value attained by $\Delta \tilde{f}_{\left|\Delta \tilde{\rho}_{\text {eff }}\right|>10}$ when the shell inertia is small compared to the core inertia. Besides, the variations 
of $\Delta \tilde{f}_{\left|\Delta \tilde{\rho}_{\text {eff }}\right|>10}$ versus $\varepsilon$ ranging from 0.06 to 6 for several realistic values of $\Delta \tilde{\rho}$, shown in insert of Fig. 6 , evidence that $\Delta \tilde{f}_{\left|\Delta \tilde{\rho}_{\text {eff }}\right|>10}$ is maximal at $\varepsilon \simeq 0.1-0.2$ and that this maximum increases with $\Delta \tilde{\rho}$.

This analysis of the width of the resonances of the core displacement amplitude and of the effective density will help to explain the dependence of the negative effective density frequency band versus the density contrast and the shell thickness, which are studied hereafter.

\section{NEGATIVE EFFECTIVE DENSITY FREQUENCY BAND}

In this section, we study in which conditions a negative effective density is actually obtained in absence of dissipation, i.e., how the frequency band $\Delta f_{\text {neg }}$ in which the effective density of a suspension of core-shell resonators is predicted to be negative (NEDFB) depends on $\Delta \tilde{\rho}$ and $\varepsilon$, still in absence of dissipation. The NEDFB is seeked to be as broad as possible in order to counterbalance for instance the effect of size dispersion of the resonators. The NEDFB is indicated in Fig. 2.

We note that the condition $\rho_{\text {eff }}<0$ is equivalent to $\left|\Delta \tilde{\rho}_{\text {eff }}\right|>1$. Correlatively, $\Delta f_{\text {neg }}=\Delta f_{\left|\Delta \tilde{\rho}_{\text {eff }}\right|>1}$. Thus we expect the dependence of $\Delta \tilde{f}_{\text {neg }}=\Delta f_{\text {neg }} / f_{\text {res }}$ versus $\Delta \tilde{\rho}$ and $\varepsilon$ to be qualitatively similar to the one of $\Delta \tilde{f}_{\left|\Delta \tilde{\rho}_{\text {eff }}\right|>10}$. The variations of $\Delta \tilde{f}_{\text {neg }}$ versus $\varepsilon$ ranging from 0.1 to 3 in the $\mu_{2}^{\prime} / \lambda_{2} \ll 1$ limit and for several realistic values of $\Delta \tilde{\rho}$ ranging from 1 to 16 , shown in Fig. 7 , evidence that $\Delta \tilde{f}_{\text {neg }}$ exhibits a maximum at $\varepsilon \simeq 0.1-0.2$ whatever $\Delta \tilde{\rho}$ (it exceeds the half of its maximum when $\varepsilon$ drops below 0.6). The larger the density contrast, the higher is the maximum of $\Delta \tilde{f}_{\text {neg }}$ at saturation. This trend has been found for all values of $\mu_{2}^{\prime} / \lambda_{2}$. As expected, the

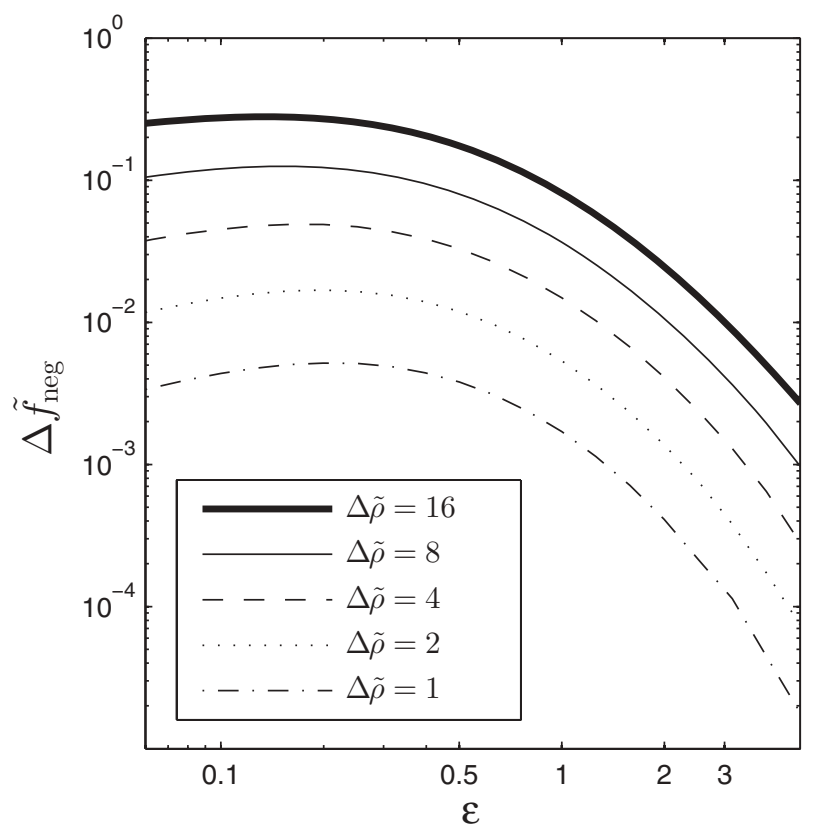

FIG. 7. Variations of the dimensionless frequency bandwidth $\Delta \tilde{f}_{\text {neg }}$ in which the effective density of the suspension of core-shell resonators is negative (NEDFB) vs the dimensionless shell thickness $\varepsilon$ for several values of the dimensionless density contrast $\Delta \tilde{\rho}$ in absence of dissipation. $\mu_{2}^{\prime} / \lambda_{2}=10^{-4}$ and $\phi=0.1$.

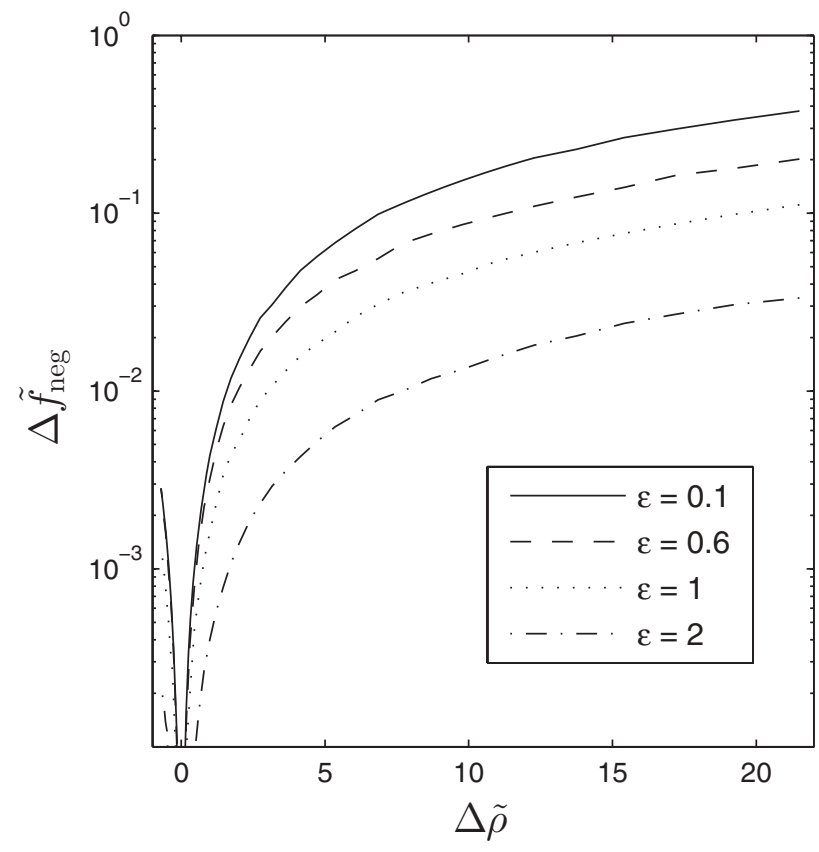

FIG. 8. Variations of the dimensionless width of the NEDFB $\Delta \tilde{f}_{\text {neg }}$ versus the dimensionless density contrast $\Delta \tilde{\rho}$ for several values of dimensionless shell thickness $\varepsilon$ in absence of dissipation. $\mu_{2}^{\prime} / \lambda_{2}=10^{-4}$ and $\phi=0.1$.

dependence of $\Delta \tilde{f}_{\text {neg }}$ versus $\varepsilon$ is qualitatively similar to the one of $\Delta \tilde{f}_{\left|\Delta \tilde{\rho}_{\text {eff }}\right|>10 \text {. }}$

Similarly, the variations of $\Delta \tilde{f}_{\text {neg }}$ versus $\Delta \tilde{\rho}$ in the $\mu_{2}^{\prime} / \lambda_{2} \ll$ 1 limit and for several values of $\varepsilon$ ranging from 0.1 to 2 , shown in Fig. 8 , evidence that $\Delta \tilde{f}_{\text {neg }}$ becomes vanishingly small as $|\Delta \tilde{\rho}|$ vanishes, whatever the value of $\varepsilon$. Again, as expected, the dependence of $\Delta \tilde{f}_{\text {neg }}$ versus $\Delta \tilde{\rho}$ is qualitatively similar to

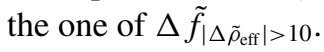

Both these results have important consequences on the design of the core-shell resonators: in order to maximize the width of the NEDFB, (i) the density contrast has to be as large as possible and (ii) the dimensionless shell thickness has to be thinner than say 0.6. When both these conditions are fulfilled, in absence of dissipation, the width of the NEDFB can attain values of the order of tens of percents of the resonance frequency for $\phi=0.1$.

\section{INFLUENCE OF DISSIPATION}

In this section, we study the influence of dissipation on the width of the NEDFB, and how it combines with the dimensions and physical properties of the resonators. The effect of dissipation is illustrated in Fig. 2, where two resonance curves are represented, one without dissipation $\left(\mu_{2}=\mu_{2}^{\prime}\right.$ real $)$ and one corresponding to a complex elastic shear modulus $\mu_{2}=\mu_{2}^{\prime}+i \mu_{2}^{\prime \prime}$ such that $\mu_{2}^{\prime \prime} / \mu_{2}^{\prime}=0.04$. Whereas $f_{\text {res }}$ is not markedly affected by dissipation, $\Delta f_{\text {neg }}$ is strongly affected. In order to quantify the effect of dissipation on the targeted negative effective density property, we define $r_{c}$ as the critical value of the ratio $\mu_{2}^{\prime \prime} / \mu_{2}^{\prime}$ beyond which the width of the NEDFB is less than the half of its value in absence of dissipation. We address the following question: how does $r_{c}$ depend on $\Delta \tilde{\rho}$ and on $\varepsilon$ ? The variations of $r_{c}$ versus $\Delta \tilde{\rho}$ for several values 


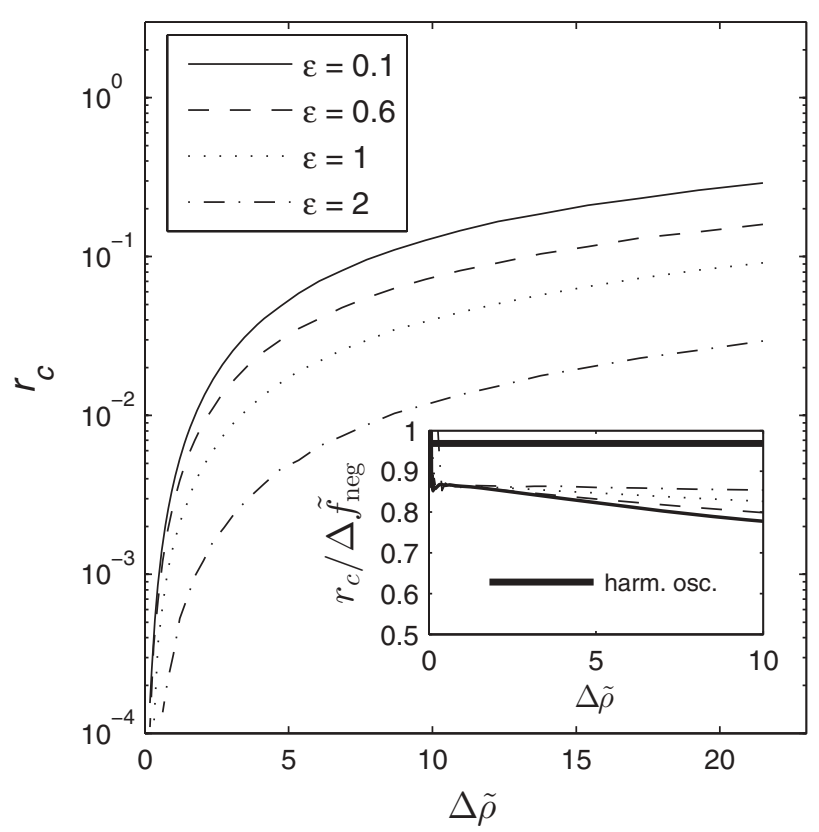

FIG. 9. Variations of the critical value $r_{c}$ of the ratio $\mu_{2}^{\prime \prime} / \mu_{2}^{\prime}$ above which the width of the NEDFB is less than half of its value in absence of dissipation vs the dimensionless density contrast $\Delta \tilde{\rho}$ for several values of the dimensionless shell thickness $\varepsilon$. $\mu_{2}^{\prime} / \lambda_{2}=10^{-4}$ and $\phi=0.1$. (Inset) Ratio of $r_{c}$ to $\Delta \tilde{f}_{\text {neg }}$ vs $\Delta \tilde{\rho}$ for several values of $\varepsilon$ compared with its value for a harmonic oscillator.

of $\varepsilon$, plotted in Fig. 9, evidence that the larger $\Delta \tilde{\rho}$, and the smaller $\varepsilon$, the larger is $r_{c}$. This means that dissipation is less perceptible the thinner is the shell and the larger is the density contrast. The conditions required for obtaining broad NEDFB in absence of dissipation appear as similar to those required for being the less affected by dissipation. More precisely, as shown in the inset of Fig. 9, which displays the variations of the ratio $r_{c} / \Delta \tilde{f}_{\text {neg }}$ versus $\Delta \tilde{\rho}$ and $\varepsilon, r_{c} / \Delta \tilde{f}_{\text {neg }}$ is actually roughly constant.

This striking fact can be qualitatively explained by considering the harmonic oscillator again. As shown above, in absence of dissipation, a harmonic oscillator forced with fixed displacement amplitude is such that $\Delta \tilde{f}_{\tilde{x}>T} \simeq T^{-1}$ in the $T \gg 1$ limit. In presence of dissipation, the displacement amplitude of a damped harmonic oscillator is such that $\tilde{x}=\left[1-\tilde{\omega}^{2}+i\left(\gamma_{2} \omega_{\text {res }} / k_{2}\right) \tilde{\omega}\right]^{-1}$, where $-\gamma_{2} \dot{x}$ is the friction force acting on the oscillator. We define here $r_{c}$ as the critical value of the ratio of dissipation to stiffness $\gamma_{2} \omega_{\text {res }} / k_{2}$ beyond which the dimensionless width of the frequency band over which $\tilde{x}$ is larger than a given threshold $T, \Delta \tilde{f}_{\tilde{x}>T}$, is less than the half of its value in absence of dissipation. It can be easily shown that $r_{c} \simeq \sqrt{15 / 16} T^{-1}$ in the $T \gg 1$ limit. Thus, in this limit, $r_{c} \simeq \sqrt{15 / 16} \Delta \tilde{f}_{\tilde{x}>T}$. This result is also found to be valid for the mass-in-mass lattice since its effective mass $m_{\text {eff }}$ satisfies $\Delta \tilde{m}_{\text {eff }}=\left(m_{3} / m_{0}\right) \tilde{\omega}^{2} \tilde{x}$. Coming back to the core-shell resonator, we note that the equivalence between the damped harmonic resonator, the damped mass-in-mass lattice, and the core-shell particle with viscoelastic shell is ensured by the correspondences $k_{2} \Longleftrightarrow \mu_{2}^{\prime}$ and $\gamma_{2} \Longleftrightarrow \omega_{\text {res }} \mu_{2}^{\prime \prime}$. Thus the sensitivity of the resonance width of a core-shell resonator to dissipation is similar to the one of a harmonic oscillator or of the mass-in-mass lattice: for all these systems, $r_{c}$ is proportional to $\Delta f_{\alpha>T}, \alpha$ being $\tilde{x},\left|\Delta \tilde{m}_{\text {eff }}\right|$ or $\left|\Delta \tilde{\rho}_{\text {eff }}\right|$. Thus the constancy of $r_{c} / \Delta \tilde{f}_{\text {neg }}$ in the case of the core-shell suspension appears as a direct consequence of the fact that a core-shell resonator behaves as a harmonic resonator. We note from the inset of Fig. 9 that the ratio $r_{c} / \Delta \tilde{f}_{\text {neg }}$ for the core-shell resonator is even close to $\sqrt{15 / 16}$, which is the value of this ratio for the harmonic resonator.

\section{CONCLUSION}

Thanks to a parametric study of the analytical model of Ref. 28 describing the mechanical response of a core-shell particle to an acoustic excitation, we have computed an abacus for the resonance frequency of these particles in the case of thin shells made of a soft solid. The first main result of this study is that the value of the first Lamé coefficient $\lambda$ of the material constituting the shell has not necessarily to be "small" for satisfying the condition of subwavelength resonator dimension at resonance. Only a "small" value of its elastic shear modulus is actually required. In addition, we have quantified the influence of the dimensions of the resonators and of the viscoelastic properties of the materials constituting the core and the shell on the resonance. In particular, we could qualitatively explain the dependence of the frequency band over which the effective density of the resonator suspension is negative on the density contrast between the core and the shell by comparing it with the behavior of a harmonic oscillator and of the mass-in-mass lattice. The second main result of this study is that the larger the density contrast between the core and the shell, and the thinner the shell, the broader is the frequency band over which the effective density of the resonator suspension is negative, but that it is practically useless to decrease the dimensionless shell thickness below 0.6 . Finally, we have shown that the dissipation is less perceptible the thinner is the shell and the larger is the density contrast, and we could explain this observation by comparison with the harmonic oscillator and the mass-in-mass lattice. According to this model, both maximization of the width of the NEDFB of such suspensions and minimization of the effect of dissipation are thus expected for large density contrasts and thin shells. These conclusions should help in designing optimal core-shell resonators to select adequate materials for constituting the core and the shell and to design innovative processes of fabrication of downsized core-shell resonators based on self-assembling or microfluidics. These results also explain why the only core-shell based LRAM exhibiting negative effective density ever produced was composed of resonators made of a lead core encapsulated in a rubber shell with small relative thickness $(\Delta \tilde{\rho}=7.9$ and $\varepsilon=0.5){ }^{7}$

\section{ACKNOWLEDGMENTS}

The authors thank Olivier Mondain-Monval and Jacques Leng for fruitfull discussions and acknowledge Bordeaux University, Région Aquitaine, CNRS, the cluster Advanced Materials in Aquitaine (GIS AMA), and ANR (METAKOUSTIK grant No. ANR2011-BS0902102) for funding and support. 
${ }^{1}$ L. Feng, X.-P. Liu, M.-H. Lu, Y.-B. Chen, Y.-F. Chen, Y.-W. Mao, J. Zi, Y.-Y. Zhu, S.-N. Zhu, and N.-B. Ming, Phys. Rev. Lett. 96, 014301 (2006).

${ }^{2}$ M.-H. Lu, C. Zhang, L. Feng, J. Zhao, Y.-F. Chen, Y.-W. Mao, J. Zi, Y.-Y. Zhu, S.-N. Zhu, and N.-B. Ming, Nat. Mater. 6, 744 (2007).

${ }^{3}$ A. Sukhovich, B. Merheb, K. Muralidharan, J. O. Vasseur, Y. Pennec, P. A. Deymier, and J. H. Page, Phys. Rev. Lett. 102, 154301 (2009).

${ }^{4}$ S. Yang, J. H. Page, Z. Liu, M. L. Cowan, C. T. Chan, and P. Sheng, Phys. Rev. Lett. 93, 024301 (2004).

${ }^{5}$ S. H. Lee, C. M. Park, Y. M. Seo, Z. G. Wang, and C. K. Kim, Phys. Rev. Lett. 104, 054301 (2010).

${ }^{6}$ M.-H. Lu, L. Feng, and Y.-F. Cheng, Mat. Today 12, 34 (2009).

${ }^{7}$ Z. Liu, X. Zhang, Y. Mao, Y. Y. Zhu, Z. Yang, C. T. Chan, and P. Sheng, Science 289, 1734 (2000).

${ }^{8}$ C. M. Park, J. J. Park, S. H. Lee, Y. M. Seo, C. K. Kim, and S. H. Lee, Phys. Rev. Lett. 107, 194301 (2011).

${ }^{9}$ N. Fang, D. Xi, J. Xu, M. Ambati, W. Srituravanich, C. Sun, and X. Zhang, Nat. Mater. 5, 452 (2006).

${ }^{10}$ S. Zhang, L. Yin, and N. Fang, Phys. Rev. Lett. 102, 194301 (2009).

${ }^{11}$ S. Zhang, C. Xia, and N. Fang, Phys. Rev. Lett. 106, 024301 (2011).

${ }^{12}$ K. M. Ho, C. K. Cheng, Z. Yang, X. X. Zhang, and P. Sheng, Appl. Phys. Lett. 83, 5566 (2003).

${ }^{13}$ Z. Yang, J. Mei, M. Yang, N. H. Chan, and P. Sheng, Phys. Rev. Lett. 101, 204301 (2008).

${ }^{14}$ Z. Yang, H. M. Dai, N. H. Chan, G. C. Ma, and P. Sheng, Appl. Phys. Lett. 96, 041906 (2010).

${ }^{15}$ V. Leroy, A. Strybulevych, J. H. Page, and M. G. Scanlon, J. Acoust. Soc. Am. 123, 1931 (2008).
${ }^{16} \mathrm{Ph}$. Lambin, A. Khelif, J. O. Vasseur, L. Dobrzynski, and B. DjafariRouhani, Phys. Rev. E 63, 06660 (2001).

${ }^{17}$ M. Hirsekorn, P. P. Delsanto, N. K. Batra, and P. Matic, Ultrasonics 42, 231 (2004).

${ }^{18}$ J. Li and C. T. Chan, Phys. Rev. E 70, 055602 (2004).

${ }^{19}$ J. Li, Z. Liu, and C. Qiu, Phys. Rev. B 73, 054302 (2006).

${ }^{20}$ Y. Ding, Z. Liu, C. Qiu, and J. Shi, Phys. Rev. Lett. 99, 093904 (2007).

${ }^{21}$ S. Guenneau, A. Movchan, G. Pétursson, and S. A. Ramakrishna, New J. Phys. 9, 399 (2007).

${ }^{22}$ H. Larabi, Y. Pennec, B. Djafari-Rouhani, and J. O. Vasseur, Phys. Rev. E 75, 066601 (2007).

${ }^{23}$ X. Ao and C. T. Chan, Phys. Rev. E 77, 025601 (2008).

${ }^{24}$ X. Zhou and G. Hu, Phys. Rev. B 79, 195109 (2009).

${ }^{25}$ Y. Wu, Y. Lai, and Z.-Q. Zhang, Phys. Rev. Lett. 107, 105506 (2011).

${ }^{26}$ Leighton, The Acoustic Bubble (Academic, San Diego, California, 1997).

${ }^{27}$ K. W. Commander and A. Prosperetti, J. Acoust. Soc. Am. 85, 732 (1989).

${ }^{28}$ Z. Liu, C. T. Chan, and P. Sheng, Phys. Rev. B 71, 014103 (2005).

${ }^{29}$ Z. Liu, C. T. Chan, and Ping Sheng, Phys. Rev. B 65, 165116 (2002).

${ }^{30}$ G. W. Milton and J. R. Willis, Proc. R. Soc. London A 463, 855 (2007).

${ }^{31}$ J. Li, K. H. Fung, Z. Y. Liu, P. Sheng, and C. T. Chan, in Physics of Negative Refraction and Negative Index Materials, Springer Series in Materials Science Vol. 98, edited by C. M. Krowne, M. Clifford and Y. Zhang (Springer, Verlag, 2007).

${ }^{32}$ H. H. Huang, C. T. Sun, and G. L. Huang, Int. J. Eng. Sci. 47, 610 (2009). 